\title{
Pour en lire plus : Un art écologique. Création plasticienne et anthropocène.
}

Maia Morel

\section{(2) OpenEdition \\ Journals}

Édition électronique

URL : https://journals.openedition.org/ere/6376

DOI : $10.4000 /$ ere.6376

ISSN : 2561-2271

Éditeur

Centr'ERE

Référence électronique

Maia Morel, «Pour en lire plus : Un art écologique. Création plasticienne et anthropocène. », Éducation relative à l'environnement [En ligne], Volume 16 - 1 | 2021, mis en ligne le 10 mars 2021, consulté le 27 mai 2021. URL : http://journals.openedition.org/ere/6376 ; DOI : https://doi.org/10.4000/ere.6376 


\section{Pour en lire plus : Un art écologique. Création plasticienne et anthropocène.}

Maia Morel

\section{RÉFÉRENCE}

Paul Ardenne (2019). Un art écologique. Création plasticienne et anthropocène. Lormont :

Éditions Le Bord de l'Eau, collection « La Muette », 277 pages. 


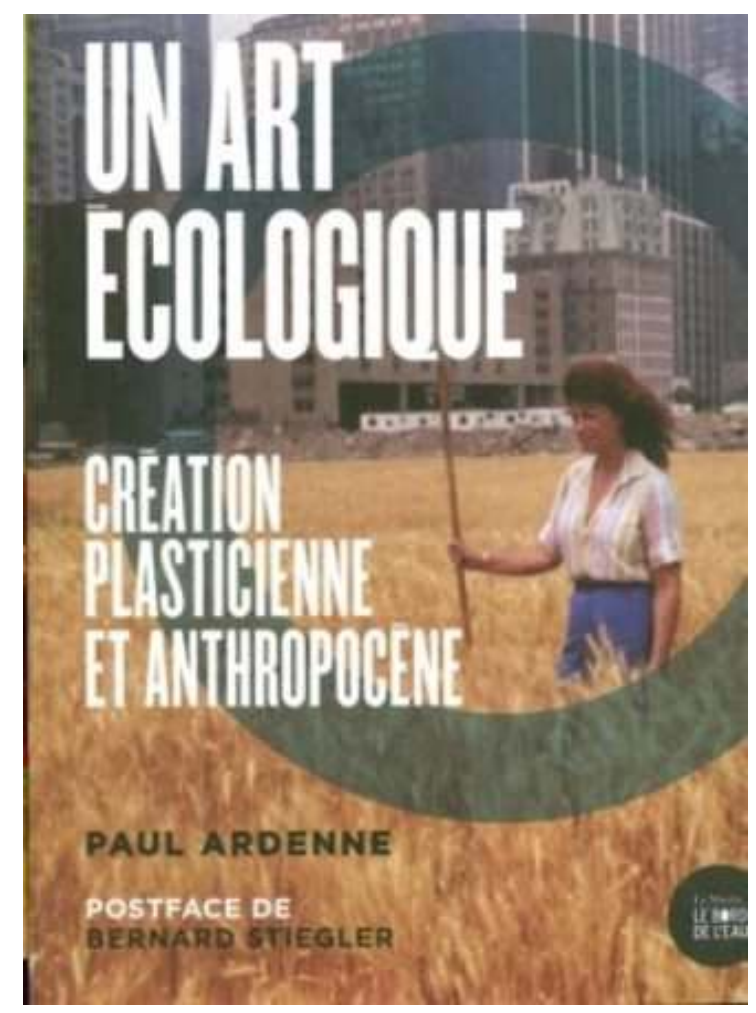

1 Ouvrir un livre de Paul Ardenne signifie toujours rencontrer une personnalité forte, une pensée structurée et des opinions bien arrêtées. Cette nouvelle édition d'Un art écologique. Création plasticienne et anthropocène, qui vise à nous présenter un «tour de table des rapports nouveaux tissés entre la création plastique et la question écologique » (p. 263), ne fait pas exception à la règle.

Bien présenté, riche de références culturelles très diverses, mais gêné parfois par une syntaxe peu fluide, le livre se présente en trois parties, précédées d'une introduction et suivies d'une brève conclusion. L'ouvrage suit une progression à la fois logique et chronologique qui montre comment l'art est petit à petit devenu un des lieux du combat écologique, en même temps que ce dernier prenait une place croissante dans l'actualité et la pensée sociale. Le fil conducteur est donc, dans un monde désormais entièrement soumis aux diktats de l'exploitation humaine (l'anthropocène), l'émergence d'« artivistes » qui, avec leurs armes propres, « celles de la représentation, de la symbolique, de l'éthique » (p. 2), tentent d'œuvrer en faveur de l'environnement.

Les objectifs de l'auteur sont à la fois descriptifs et prescriptifs :

- Descriptifs : «cet essai [...] se prévaut d'une ambition [...] d'abord documentaire : indexer des positions d'alerte, des comportements vigiles, des attitudes où solidarité, fraternité, humanisme prennent une place décisive et se traduisent en formes, en artefacts plastiques dont le thème est la préservation de l'humain et de son milieu de vie » (p. 12).

- Prescriptifs : « rendre compte d'une préoccupation toujours plus torturante à mesure que le temps passe, préoccupation que hante cette double question, la seule qui vaille à présent: comment vivre dans un monde malade sans y simplement survivre et, de ce monde malade, que faire pour qu'il recouvre la santé ? » (ibidem).

4 La première partie du livre, intitulée prendre un bain de nature, nous convie à découvrir comment l'art a, d'une certaine manière, pris conscience de la nature après la Seconde Guerre mondiale. Si, en effet, la fin du $19^{e}$ siècle a fait sortir l'artiste de son 
atelier, ce n'était que pour reconstituer celui-ci à l'extérieur, le but principal du travail artistique restant la représentation, qui implique la mise à distance du sujet. Un siècle plus tard, «l'heure de l'immersion a sonné » (p. 21). L'artiste découvre le fait naturel, il jette sur son environnement un autre regard, plus curieux, plus respectueux. Certains mêmes expérimentent une véritable fusion avec la nature qui prend une dimension métaphysique, ou plus souvent existentialiste: "L'important n'est pas tant de communiquer, que d'exister" (p. 38). Exemples à l'appui, l'auteur nous montre comment la nature devient une véritable partenaire de l'acte créateur. Avec une restriction toutefois, et de taille: ce que cherchent les artistes en investissant les espaces naturels, c'est d'abord un nouveau champ de création; leur travail reste avant tout esthétique (p. 48) et la préoccupation écologique n'y est pas présente (voir notamment l'exemple de Christo à la p. 49). Bref, ici « [o]n utilise, plus que l'on célèbre. On ne s'inscrit en rien dans l'inquiétude environnementale » (p. 53).

5 Il faudra quelque temps encore avant que s'amorce une évolution vers l'éco-création ( $2^{\text {ème }}$ partie). Au début des années 1970, c'est la pollution (et plus tard le "réchauffement climatique ») qui sert de déclencheur à la prise de conscience. Plusieurs voies sont esquissées pour aller vers un rapport plus harmonieux et moins destructeur à la nature. Toutefois, les actions sont dérisoires face au développement industriel. D'ailleurs, à l'inverse d'autres formes artistiques (cinéma, BD), les arts plastiques ne sont pas de ce combat: s'ils apprécient les thématiques postapocalyptiques, cela reste pour en faire des représentations maniérées et esthétisantes qui n'ont pas vocation à porter un quelconque message. Pourquoi cette absence de prise de position? Peut-être parce que la perspective est trop effrayante, suggère Paul Ardenne.

6 De fait, une étape supplémentaire doit être franchie : "être "écologique" commande [...] de se fondre [dans l'élément naturel] et, si catastrophe environnementale il y a - et il y a à présent, en cours -, d'engager un processus de riposte» (p. 67). Bref, il faut se faire militant dans un monde à l'agonie qui, que nous le voulions ou pas, est à l'aube de mutations dramatiques. Il s'agit également de renverser un ordre matériel et symbolique qui, depuis toujours, a fait de l'homme le maître de la Nature. Comme l'écrit en 1969 Lee Ufan, membre de Mono-Ha (groupe japonais avant-gardiste actif autour de 1970), « il faut que nous sachions observer le monde tel quel et non que nous le transformions par le truchement d'une représentation qui le dresse contre l'homme » (p. 78). L'artiste doit désormais faire partie de la nature : ainsi peut naître l'« éco-œuvre d'art ». «La nature est tout à la fois un être, une coprésence, une force. Travailler en elle, oui. Mais il est mieux encore de travailler avec elle, d'en faire un partenaire actif de la création artistique.» (p. 85). Ce partenariat qui unit la Terre et l'artiste prend de multiples formes que l'auteur explore de manière chronologique depuis la fin du $20^{\mathrm{e}}$ siècle. Elles ont pour point commun d'être une réflexion sur notre lien à la nature et d'insister sur les interactions multiples existant entre les différentes composantes de celle-ci et qui en font un tout.

7 Ce travail de réflexion rejoint par ailleurs, nous rappelle Paul Ardenne, de vieux mythes édéniques : «le monde est un bien, il est offert aux créatures qui le peuplent comme un présent à respecter» (p.95). Débarrassé de ses connotations religieuses, ce thème naturiste est pour l'auteur à l'origine de l'émergence, dès la fin du $18^{\mathrm{e}}$ siècle, d'une thématique du paysage et de la «nature sans l'homme » qui prend une place de plus en plus importante dans la peinture - comme en témoignent les écoles paysagistes 
américaines - ainsi que dans de multiples formes de représentations (photographie, télévision...) jusqu'à notre époque.

Déclaration d'amour à la nature, dévotion, admiration, « désir de proximité hautement sensible avec l'élément naturel » (p. 108), c'est un véritable culte qui s'est peu à peu construit, à un détail près - mais il est d'importance - qui est que ce monde primitif dont nous avons la nostalgie n'existe plus, parce que nous l'avons irrémédiablement détruit. Encore faut-il en avoir conscience: "À contresens de la valorisation de l'exaltation, l'honnêteté artistique réside plus sûrement dorénavant, plus que dans sa négation, dans l'enregistrement de la catastrophe écologique»(p.112). Dure réalité que les photographes seront les premiers à saisir dans la deuxième moitié $\mathrm{du} 20^{\mathrm{e}}$ siècle.

Cela veut-il dire pour autant que l'écologie aurait partie gagnée ? Certes non, car ce qui domine en réalité c'est avant tout la récupération du label « vert », et cela pour servir les mêmes buts que par le passé.

Toutefois, des efforts réels méritent d'être soulignés et c'est ce que fait l'auteur en nous livrant une intéressante analyse de l'architecture du $21^{\mathrm{e}}$ siècle (domaine sensible, car il met en jeu de nombreux paramètres techniques, juridiques et sociaux) et de son évolution. Limitée mais réelle, celle-ci marque le passage d'un idéal d'architecture somptuaire vers un idéal d'éco-architecture (p. 118 et suivante.).

11 Quant à l'artiste, que peut-il faire concrètement pour apporter sa pierre au travail écologique? Le défi est complexe. De plus, ne risque-t-on pas de confondre ici action artistique et action de l'artiste (qui agit comme n'importe quel citoyen)? Non, répond Paul Ardenne, en vertu du concept duchampien que « l'"art", c'est tout ce que l'artiste a décidé de décréter tel» (p.122). Ce qui fait la différence, c'est donc l'engagement, l'impact du geste, et le privilège que s'attribue l'artiste d'être une personne différente.

Dans cet univers où il devient essentiel de faire en sorte que chacun de nos gestes génère un résultat environnemental positif (p.134), plusieurs pratiques artistiques se sont ainsi développées : utiliser des matériaux de récupération, par exemple, ou tout simplement témoigner de son désarroi, voire chercher à rencontrer une dernière fois les quelques lieux qui peuvent conserver une apparence de pureté originelle.

C'est que la Maison Terre se meurt et nombreux sont les artistes qui s'en alarment et cherchent à mettre en avant la thématique de la dégradation des milieux naturels.

S'acheminerait-on pas à pas vers l'art utile ? Tel est le sujet qu'aborde la $3^{\text {ème }}$ partie de l'ouvrage.

Le constat est là, brutal : notre maison commune est irrémédiablement compromise et tout retour en arrière est illusoire. Que faire, et comment? car «il revient aussi au champ de l'art, à sa mesure et avec ses moyens propres, de réagir » (p. 167). Comme pour repartir de zéro, de nombreux plasticiens explorent le thème de la maison, une maison que l'on veut écologiquement parfaite, et pourquoi pas une cabane, à l'image de ce refuge élémentaire qu'Henri David Thoreau s'était construit près de l'étang de Walden, dans le Massachusetts, en 1845 (p. 158 et suiv.). D'autres s'engagent, pour l'Amazonie par exemple, appelant à un retour à la nature originelle. Au point de rencontre de ces deux préoccupations, on verra aussi des artistes créer d'innombrables habitats précaires, de récupération, destinés à des publics marginalisés ou en recherche, visant tous un retour vers l'élémentaire, le fondamental, jusqu'à se fondre dans la terre s'il le faut, comme Tixador et Poncheval qui progressent dans une galerie à 1 mètre 50 de profondeur, pendant 20 jours, au rythme d'1 m par jour (p. 172 et suiv.), 
ou - pourquoi pas? - jusqu'à s'inclure dans la pierre, tel Timm Ulrichs qui passera 7 heures, nu, dans une cavité qu'il a fait creuser dans un énorme bloc de pierre (p. 175-177). Tous recherchent le contact direct avec la nature, certes, mais cela ne suffit pas. Encore faut-il que l'action artistique intègre une dimension collective : « un éco-art doit forcément avoir en vue l'intérêt public, et se constituer comme formule ouverte» (p. 177). Sans engagement, sans militantisme, il n'y a pas d'éco-art, "[à] savoir, plus et autrement mieux qu'une "forme" plastique, un combat, une intervention déterminée dans l'espace du réel et pour l'amélioration du réel » (p. 178). Quelques exemples viennent à l'appui de cette position, comme celui de l'événement Art et écologie. 1 temps - 6 lieux, organisé au Québec en 1983, qui est à cet égard éloquent : nous sommes ici dans le domaine de l'éthique et à l'opposé de toute considération mercantile.

$\mathrm{Au}$ demeurant, cet activisme artistique écologique, fruit d'une multitude de petites interventions éco-éthiques qui promeuvent un « processus de fraternisation entre l'art et l'utilité sur fond de non-dégradation» (p.184), pose au fond le problème de la définition même de l'art. Ne perd-il pas son âme en devenant utile? Pas aux yeux de Paul Ardenne, pour qui «l'éco-art d'aujourd'hui doit nourrir la réflexion future » (p. 191).

17 L'objectif est désormais "[u]ne forme d'art qui est une formule d'avertissement » (p. 193), qui se veut responsable et vise à soutenir une prise de conscience écologique essentielle devant l'insupportabilité de la dégradation de l'environnement. Sans doute cette démarche s'accomplit-elle parfois au prix de la dimension esthétique des œuvres mais elle apparait comme une option, ni pire ni meilleure que d'autres, que le créateur est libre de choisir et qui fait de lui un véritable lanceur d'alerte.

À cette étape de son parcours, Paul Ardenne s'arrête ici, comme en point d'orgue, sur la thématique de l'arbre. Riche de toute une tradition symbolique et artistique l'arbre présente pour l'éco-artiste la particularité d'être à la fois «victime (déforestation) et solution (élimination du carbone)»(p. 219), d'où l'attention privilégiée dont il est l'objet. Comme à chaque fois que l'auteur s'attarde sur un point précis, il nous livre une analyse fine et éclairante, portant en l'occurrence sur les thèmes de l'arbre et de la forêt et sur leur évolution, jusqu'aux innombrables créations de l'éco-art contemporain.

19 Travailler avec des éléments naturels n'équivaut toutefois pas ipso facto à faire de l'écoart. Paul Ardenne le rappelle : beaucoup d'œuvres sont médiocres, conventionnelles, fruit d'un souci de «faire vert» qui est avant tout une mode. L'éco-art n'est pas l'apanage de tous: "Pas d'éco-art qui vaille si celui-ci en passe par des modes conventionnels d'expression» (p. 237). Surtout, l'essentiel est ailleurs : «L'éco-œuvre réussie [...] est invariablement une affaire de responsabilité, de civisme, d'éthique " (p. 239). L'écologie n'est qu'une partie d'une écosophie plus globale qui inclut également des enjeux sociétaux, même s'il faut pour cela s'imposer quelques sacrifices. Ainsi en va-t-il de l'art du recyclage, ou de l'art du soin, du réconfort. Pour les entreprises éco-artistiques éthiques, "[1]'important [...] c'est de signifier en premier lieu un état de fait problématique et matière à travailler à le réduire » (p. 254). Là est l'espoir d'un authentique éco-art, de ce que Paul Ardenne définit dans sa brève conclusion comme un anthropocènart "démonstratif, militant, exemplaire et pas seulement illustratif » (p. 266). 
20 Le cheminement de l'auteur d'Un art écologique. Création plasticienne et anthropocène se présente donc comme double : histoire de l'art et histoire de la pensée se soutiennent et s'illustrent mutuellement, pour constituer ce qui a tout d'un manifeste, c'est-à-dire d'un texte militant. Son propos donne l'impression que c'est l'avertissement écologique qui structure le raisonnement : sans apporter d'éléments vraiment nouveaux, il pourra séduire par sa véhémence, par la colère sincère qu'on y sent et par l'ironie à laquelle il recourt. Toutefois, ce sont surtout les références artistiques qui retiendront le lecteur : son argumentaire, construit avec beaucoup de soin et de méthode, s'appuie en effet sur une connaissance qu'on pourrait dire encyclopédique (mais toutefois sélective) de l'art et particulièrement de son développement depuis la seconde moitié du $20^{\text {ème }}$ siècle. C'est là une des principales qualités de l'ouvrage qui est jalonné de renvois à des artistes et à des œuvres qui, à chaque fois, font l'objet d'une présentation claire et d'une analyse habilement menée qui en met en valeur l'intérêt.

21 La lecture du texte pourra parfois être entravée par une syntaxe complexe et par l'abondance des allusions culturelles. On appréciera aussi diversement un usage de mots anglais, souvent suivis de leur traduction en français, qui semble plus relever de l'entre-soi qu'être véritablement « useful» (pour employer un terme que l'auteur affectionne).

Il reste que Paul Ardenne s'est à juste titre mérité une réputation de penseur de premier plan dans le domaine des arts plastiques. Qu'il mette sa culture et ses capacités d'analyse au service de la cause écologique est une démarche dont on ne peut que se réjouir, comme on ne peut que saluer le talent avec lequel il met en évidence le rôle d'éveil de la conscience qui peut revenir à l'art dans ce combat.

\section{AUTEUR}

\section{MAIA MOREL}

Maia Morel est professeure agrégée à l'Université de Sherbrooke. Ses intérêts de recherche couvrent principalement deux axes : le premier vise les liens entre l'enseignement des arts et les domaines généraux de formation avec tous les avantages éducatifs que ce binôme peut offrir à l'ÉRE et au "mieux vivre ensemble". Un deuxième axe vise l'intégration de la dimension culturelle dans l'enseignement et concerne la formation des futur-e-s enseignant-e-s au rôle de « passeurs culturels ». 